\title{
Semantic SEM Image Segmentation of Concrete with Contextual Labels
}

Authors: Peter Bajcsy ${ }^{1 *}$, Steve Feldman ${ }^{2}$, Mary Brady ${ }^{1}$ and Kenneth Snyder ${ }^{2}$

1. Software and Systems Division, Information Technology Laboratory, NIST, Gaithersburg, MD, USA.

2. Materials and Structural Systems Division, Engineering Laboratory, NIST, Gaithersburg, MD, USA.

*Corresponding author: peter.bajcsy@nist.gov

Alkali-silica reaction (ASR), one of the most destructive and costly causes of concrete deterioration worldwide results from the complex interaction between highly alkaline pore solution of concrete and certain forms of reactive silica found in many types of concrete aggregate. Alkali-silica reaction results in the formation of a hygroscopic gel that expands under high relative humidity conditions $(>80 \% \mathrm{RH})$ resulting in volume increase and generation of tensile stresses which leads to concrete cracking. Understanding ASR is particularly important in hydraulic structures (dams) and nuclear power plants where early detection and measurement is critical for safety concerns, and for maintenance, repair, and relicensing considerations. Scanning Electron Microscopy (SEM) is one of the well-established imaging tools for the studying ASR in concrete, but image analysis methods to quantify the degree of reaction and its progression over time, and indeed to predict future expansion, are limited. This is in part because of limitations with image segmentation in reactive concrete aggregates with complex lithologies and textures.

We approached the segmentation problem by applying Convolutional Neural Network $(\mathrm{CNN})$ based methods to predict the damage classes due to ASR at each SEM image pixel. The target damage classes were defined as paste damage, aggregate damage, air voids, and no damage. The challenges in using the CNN-based methods lie in preparing large numbers of training labeled images while having limited resources for generating high-quality pixel-level SEM image annotations. To address these challenges, we leveraged the NIST Web Image Processing Pipeline (WIPP) system [1] and ImageJ/Fiji software tools [2] to prepare strong (pixel-level) and weak (rectangular- and circular-level) image annotations. In addition, we custom-designed image processing algorithms to estimate the damage type label and the contextual classes that can assist in assigning the target damage classes. The damage type label can be estimated by simple thresholding and region-based filtering. The contextual classes include paste type, aggregate type (quartzite, feldspars, volcanics), and iron oxides. They are estimated by translating rulebased descriptions into image processing workflows. Once the contextual class labels are assigned to pixels, the damage classes are derived by re-assigning the damage pixels into sub-classes based on the shape and location of damage labels with respect to contextual classes. The combinations of annotation tools, strong and weak image annotations, and automated detection of contextual classes following rulebased descriptions enabled us to generate strong annotations for 1630 fields of views (FOVs). The annotation quality was evaluated against manually prepared 12 FOVs with strong annotations from raw images, 31 FOVs with weak and strong annotations from a raw mosaic image, and 106 FOVs with weak annotations from an overlay of raw images and damage masks. The manual preparation per FOV varied depending on the content complexity (about $2-4 \mathrm{~h} / \mathrm{FOV}$ x 12 FOVs, 7-14 min/FOV x 31 FOVs, 15-20 $\mathrm{min} / \mathrm{FOV} \times 106$ FOVs). To derive target classes by using the custom-designed image processing algorithms took around 55s/FOV (code optimization and parallelization have not been explored). The quality of annotations was measured using the Dice index for strong reference annotations and using the confusion matrix for weak reference annotations. We plan to disseminate the 1630 FOVs with pixellevel annotations together with the trained $\mathrm{CNN}$ models. 


\section{References:}

[1] P Bajcsy, J Chalfoun and M Simon in "Web Microanalysis of Big Image Data", (Springer-Verlag International). http://www.springer.com/gp/book/9783319633596.

[2] CT Rueden, J Schindelin and MC Hiner et al., BMC Bioinformatics 18 (2017), p. 529. PMID 29187165, doi:10.1186/s12859-017-1934-Z

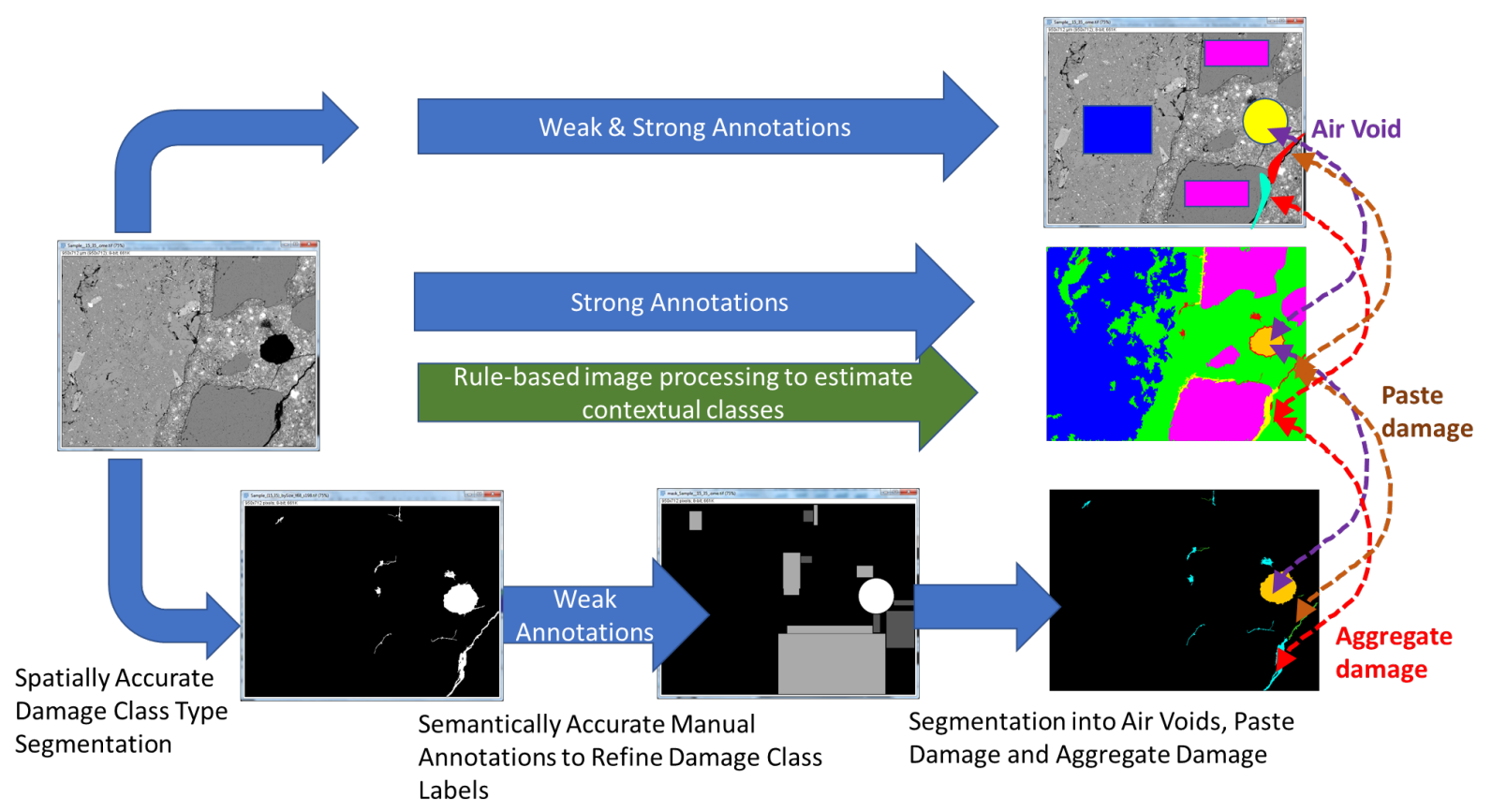

Figure 1. Overview of preparing a large quantity of high quality SEM image annotations using a combination of strong and weak annotations, as well as rule-based image processing to estimate contextual classes. 